\title{
Colour confinement and massive gluons
}

\author{
Masud Chaichian ${ }^{* \dagger}$ \\ Department of Physics, University of Helsinki, and Helsinki Institute of Physics, \\ P.O. Box 64, FI-00014 Helsinki, Finland. \\ E-mail: masud.chaichian@helsinki.fi
}

Colour confinement is one of the central issues in QCD so that there are various interpretations of this feature. In this paper we have adopted the interpretation that coloured particles are not subject to observation just because coloured states are unphysical in the sense of BRST cohomology. It is shown that there are two phases in QCD distinguished by different choices of the gauge parameter. In one phase, called the "confinement phase", colour confinement is realized and gluons turn out to be massive. In the other phase, called the "deconfinement phase", colour confinement is not realized, but the gluons remain massless.

Quarks, Strings and the Cosmos - Héctor Rubinstein Memorial Symposium

August 9-11 2010

AlbaNova, Stockholm, Sweden

${ }^{*}$ This work is dedicated to the memory of my teacher and a lifelong friend Héctor Rubinstein with the hope that its subject will stay alive for ever, as my feelings towards the Dear friend. I believe that the co-author of the work, Kazuhiko Nishijima, had he still been among us, would have also liked to dedicate a work to the same purpose.

${ }^{\dagger}$ Work originally done in collaboration with the late Professor Kazuhiko Nishijima, Department of Physics, University of Tokyo, 7-3-1 Hongo, Bunkyo-ku, Tokyo 113-0033, Japan. 


\section{Introduction}

Strong interactions of hadrons are considered to be governed by QCD dealing with the interactions of quarks and gluons. It is a gauge theory based on the colour $S U(3)$ group and a strongly interacting particle belongs to an irreducible representation of this group, for instance, quarks and gluons belong to the colour triplet and octet representations, respectively. It so happens, however, that those particles that belong to non-singlet representations are not subject to direct observations and this property is referred to as colour confinement. Thus both quarks and gluons are unobservable, and the only observable particles are colour singlet composite particles called hadrons.

Experimentally, we can study the properties of quarks and gluons only through hadrons. In order to study hadron-hadron interactions theoretically we shall first illustrate the problem by a similar one in QED. The interaction between two charged particles is governed by Coulomb's law, but the interaction between two electrically neutral particles is represented by the van der Waals potential,

$$
V_{v d W}(r) \propto r^{-6} .
$$

This shows that the electric fields generated by neutral systems penetrate into the vacuum without any sharp cut-off.

In QCD we can elucidate the dynamical properties of colour-neutral hadrons with reference to dispersion relations. For the scattering of hadrons, for instance, they remain applicable provided that confinement excludes quarks and gluons from the physical intermediate states appearing in the unitarity conditions. This is precisely the condition for colour confinement. As a typical example, let us consider nucleon-nucleon scattering, then the potential between them is given by the pole contribution in the crossed channels. The least massive hadron that can be exchanged between them is the pion, and the resulting interaction is represented by the Yukawa potential,

$$
V_{Y}(r) \propto \frac{\exp (-\mu r)}{r}
$$

where $\mu$ denotes the pion mass.

In comparison with the van der Waals force in QED we recognize that the flux of the colour gauge field emerging from colour singlet nucleons cannot penetrate into the confining vacuum beyond a certain range thereby leaving no trace of long-range forces and that the penetration depth is given by the pion Compton wave length. Thus we recognize a similarity between the Yukawa mechanism for generating nuclear forces and the Meissner effect in the type II super conductors. This similarity strongly suggests that the vacuum allows penetration of the flux of the colour gauge field generated by hadrons only by a finite length.

Thus we many point out two salient features of QCD on the basis of the experimental properties of hadrons: i) colour confinement, ii) finite penetration length of the fluxes of the colour gauge field.

The first one, colour confinement, has been elucidated in [1-8] and we shall concentrate ourselves on the second problem in the present article. Actually, we interpret ii), finite penetration length, as an evidence for massive gluons and we shall show that realization of colour confinement as interpreted in [1-8] automatically leads to massive gluons. This is the main subject of this paper. 
In Sec. 2 we shall recapitulate the arguments for colour confinement based on BRST invariance [11] and asymptotic freedom $[9,10]$. In Sec. 3 we shall show that gluons turn out to be massive when colour confinement is realized. In Sec. 4 we show that confinement is realized only in the gauges in which $Z_{3}^{-1}=0$ is realized. This is referred to as the confinement phase. On the other hand, gluons remain massless in the other gauges in which the above condition is not met, and this case is referred to as the deconfinement phase.

\section{BRST Invariance and Colour Confinement}

In this section we shall recapitulate the essence of the interpretation of colour confinement that has been developed in a series of articles [1-8].

In a covariant quantization of gauge fields introduction of indefinite metric is indispensable. Thus the resulting state vector space $\mathscr{V}$ involves unphysical states of indefinite metric and we have to find a criterion to select physical states out of $\mathscr{V}$. For this purpose we employ the Lorentz condition in QED as a subsidiary condition, but it is more involved in non-Abelian gauge theories. In what follows we shall confine ourselves to QCD, and in order to fix the notation we start from its Lagrangian density in the metric $g_{\mu \nu}=\operatorname{diag}(1,-1,-1,-1)$

$$
\mathscr{L}=\mathscr{L}_{i n v}+\mathscr{L}_{g f}+\mathscr{L}_{F P}
$$

where

$$
\begin{aligned}
& \mathscr{L}_{i n v}=-\frac{1}{4} F^{\mu v} \cdot F_{\mu v}+i \bar{\psi}\left(\gamma^{\mu} D_{\mu}-m\right) \psi, \\
& \mathscr{L}_{g f}=-A^{\mu} \cdot \partial_{\mu} B+\frac{\alpha}{2} B \cdot B, \\
& \mathscr{L}_{F P}=-i \partial^{\mu} \bar{c} \cdot D_{\mu} c
\end{aligned}
$$

in the customary notation [1-8]. We have suppressed the colour and flavor indices in (2.2). The second Lagrangian density (2.2b) is the gauge-fixing term in which $\alpha$ denotes the gauge parameter and $B$ the Nakanishi-Lautrup auxiliary field. The last one (2.2c) is the Faddeev-Popov ghost term, and the hermitian scalar fields $c$ and $\bar{c}$ are anticommuting and are called Faddeev-Popov (FP) ghost fields. The local gauge invariance is respected only by the first term (2.2a) but not by the other two, (2.2b) and (2.2c), introduced for the purpose of quantization. The total Lagrangian is invariant, however, under the global BRST transformations [11] defined below.

\section{BRST transformations}

Let us consider an infinitesimal gauge transformation of the gauge and quark fields and replace the infinitesimal gauge function either by $c$ or $\bar{c}$. They define two kinds of BRST transformations denoted by $\delta$ and $\bar{\delta}$, respectively.

$$
\begin{array}{ll}
\delta A_{\mu}=D_{\mu} c, & \bar{\delta} A_{\mu}=D_{\mu} \bar{c}, \\
\delta \psi=i g(c \cdot T) \psi, & \bar{\delta} \psi=i g(\bar{c} \cdot T) \psi .
\end{array}
$$

where the matrix $T$ is introduced in the covariant derivative of $\psi$ as

$$
D_{\mu} \psi=\left(\partial_{\mu}-i g T \cdot A_{\mu}\right) \psi
$$


For the auxiliary fields $B, c$ and $\bar{c}$ local gauge transformations are not even defined, but their BRST transformations can be introduced by requiring the invariance of the local Lagrangian density, namely,

$$
\mathscr{L}=\bar{\delta} \mathscr{L}=0 .
$$

We shall not write them down explicitly, however, since they are not relevant to the following arguments. Noether's theorem states that the BRST invariance of the Lagrangian density amounts to two conserved BRST charges denoted by $Q_{B}$ and $\bar{Q}_{B}$. They satisfy

$$
\delta \phi=i\left[Q_{B}, \phi\right]_{\mp}, \quad \bar{\delta} \phi=i\left[\bar{Q}_{B}, \phi\right]_{\mp},
$$

where we choose the $-(+)$ sign, when the field $\phi$ is of an even (odd) power in the ghost fields $c$ and $\bar{c}$.

Equations of motion for the gauge field can be expressed with the help of BRST transformations as $[1-8]$

$$
\partial^{\mu} F_{\mu v}+g J_{v}=i \delta \bar{\delta} A_{v}
$$

where $J_{v}$ denotes the colour current density and $g$ the gauge coupling constant. It is worth noting that all three terms in Eq. (2.8) are divergenceless separately, in particular

$$
\partial^{v}\left(i \delta \bar{\delta} A_{v}\right)=0
$$

The BRST charges are hermitian and nilpotent, for example,

$$
Q_{B}^{\dagger}=Q_{B}, \quad Q_{B}^{2}=0 .
$$

The nilpotency implies introduction of indefinite metric and a physical state $|f\rangle$ is defined by the constraint

$$
Q_{B}|f\rangle=0,|f\rangle \in \mathscr{V} .
$$

The set of physical states including the vacuum state $|0\rangle$ forms the physical subspace of $\mathscr{V}$ denoted by $\mathscr{V}_{\text {phys }}$,

$$
\mathscr{V}_{\text {phys }}=\left\{|f\rangle: Q_{B}|f\rangle=0,|f\rangle \in \mathscr{V}\right\} .
$$

Then the S matrix is BRST invariant and satisfies

$$
\delta S=i\left[Q_{B}, S\right]=0,
$$

so that the physical subspace $\mathscr{V}_{\text {phys }}$ is an invariant subspace of the $\mathrm{S}$ matrix.

Furthermore, we introduce a subspace of $\mathscr{V}$ called the daughter subspace $\mathscr{V}_{d}$ defined by

$$
\mathscr{V}_{d}=\left\{|f\rangle:|f\rangle=Q_{B}|g\rangle,|g\rangle \in \mathscr{V}\right\} .
$$

Then because of the nilpotency of $Q_{B}, \mathscr{V}_{d}$ is a subspace of $\mathscr{V}_{\text {phys }}$,

$$
\mathscr{V}_{d} \subset \mathscr{V}_{\text {phys }},
$$

and we introduce the Hilbert space $\mathscr{H}$ by

$$
\mathscr{H}=\mathscr{V}_{\text {phys }} / \mathscr{V}_{d}
$$


We may compare $\mathscr{V}_{\text {phys }}, \mathscr{V}_{d}$ and $\mathscr{H}$ to closed forms, exact forms, and cohomology in Cartan's algebra and (2.16) may be called the BRST cohomology [3, 13, 14].

When the quark and gluon states are not physical, they are unobservable and hence confined. Thus the problem of colour confinement reduces to demonstration of the conditions

$$
\left.Q_{B}|q u a r k\rangle \neq 0, \quad Q_{B} \mid \text { gluon }\right\rangle \neq 0 .
$$

In a series of papers [1-8] it has been shown that the criterion for colour confinement takes a simple form

$$
C=0
$$

where the constant $C$ is defined by

$$
\partial^{v}\left\langle i \delta \bar{\delta} A_{v}^{a}(x), A_{j}^{b}(y)\right\rangle=i \delta_{a b} C \partial_{j} \delta^{4}(x-y) \quad(j=1,2,3) .
$$

Here and in what follows $\langle\cdots\rangle$ denotes the vacuum expectation value of the time-ordered product.

Furthermore, with the help of the renormalization group and asymptotic freedom [1 - 5, 14], it has been shown that the conditions (2.18) follows from

$$
Z_{3}^{-1}=0,
$$

where $Z_{3}$ is the renormalization constant of the colour gauge field.

It has already been shown by Oehme and Zimmermann [15] without reference to perturbation theory that the above condition is satisfied in the Landau gauge for $N_{f}<10$ on the basis of RGE and asymptotic freedom. It is true that the anomalous dimensions in RGE are evaluated in perturbation theory, but even then some results are valid beyond perturbation theory thanks to asymptotic freedom. Depending on the nature of the objects RGE provides results at different levels of precision, namely, (1) approximate or semi-perturbative, or (2) exact. For instance, evaluation of the coefficient functions in the operator product expansion falls into the first category, whereas examination of some global properties of the theory falls into the second category. In fact, asymptotic freedom itself follows from the negative beta function evaluated in the lowest order, but this concept of asymptotic freedom is considered to be valid beyond perturbation theory. Likewise, the condition (2.20) for colour confinement falls into the second category. This condition has been derived from unbroken non-abelian gauge symmetry and asymptotic freedom related to the high energy behavior of the quark-gluon system. When $Z_{3}^{-1}$ is expressed as the integral of the Lehmann spectral function of the gluon propagator over the entire energy region, however, Eq. (2.20) indicates a delicate balance of the contributions from all energy regions including both ultraviolet and infrared. In this sense this condition gives an indirect constraint on the infrared behavior of this system. This should be contrasted with Wilson's area law in which the low-energy or long-distance behavior plays a dominant role in explaining quark confinement. It is also worth emphasizing that as a consequence of the above condition all the particles belonging to non-singlet representations of the colour group are confined for the same cause.

\section{Pole Structure of Green's Functions and Massive Gluons}

In this section we shall show that gluons turn out to be massive when the condition for colour confinement (2.18) is satisfied. For this purpose we start form Eq. (2.8). This equation has been 
given in the unrenormalized form so that we put the script (0) to unrenormalized expressions and rewrite (2.8) as

$$
\partial^{\mu} F_{\mu \nu}^{(0)}+g_{0} J_{v}^{(0)}=i \delta \bar{\delta} A_{v}^{(0)} .
$$

Then we study its relationship to the renormalized version, and in order to facilitate understanding of the nature of the problem we start from its abelian version or QED, namely,

$$
\partial^{\mu} F_{\mu \nu}^{(0)}+e_{0} J_{v}^{(0)}=-\partial_{v} B^{(0)}
$$

The multiplicative renormalization of fields and parameters relevant to this equation can be summarized as

$$
\begin{gathered}
A_{\mu}^{(0)}=Z_{3}^{1 / 2} A_{\mu}, \quad B^{(0)}=Z_{3}^{-1 / 2} B, \quad J_{v}^{(0)}=J_{v}, \\
e_{0}=Z_{3}^{-1 / 2} e, \quad \alpha_{0}=Z_{3} \alpha .
\end{gathered}
$$

The renormalized version of Eq. (3.2) is given by

$$
\partial^{\mu} F_{\mu v}+e \tilde{J}_{v}=-\partial_{v} B
$$

where

$$
e \tilde{J_{v}}=Z_{3}^{-1}\left[e J_{v}+\left(1-Z_{3}\right) \partial_{v} B\right] .
$$

It so happens that both $\partial^{\mu} F_{\mu \nu}$ and $\partial_{v} B$ are multiplicatively renormalized but with different multiplicative factors. This mismatch forces to introduce operator mixing for renormalization [16]. Since both $\partial^{\mu} F_{\mu \nu}$ and $\partial_{v} B$ are finite operators so must be $\tilde{J}_{v}$ too.

Essentially the same situation takes place in QCD, and the renormalized version of (3.1) is given by

$$
\partial^{\mu} F_{\mu \nu}^{a}+g \tilde{J}_{v}^{a}=i \delta \bar{\delta} A_{v}^{a},
$$

where $\tilde{J}_{v}^{a}$ is a linear combination of $J_{v}^{a}$ and $i \delta \bar{\delta} A_{v}^{a}$. The space integral of $J_{0}^{a}$ gives the colour charge $Q^{a}$.

$$
Q^{a}=\int d^{3} x J_{0}^{a}(x),
$$

satisfying the commutation relations of the colour SU(3) algebra,

$$
\left[Q^{a}, Q^{b}\right]=i f_{a b c} Q^{c}
$$

With the help of Eq. (3.7) we can write down an equation for two-point Green's functions of the form

$$
\left\langle\partial^{\lambda} F_{\lambda \mu}^{a}(x), A_{v}^{b}(y)\right\rangle+\left\langle g \tilde{J}_{\mu}^{a}(x), A_{v}^{b}(y)\right\rangle=\left\langle i \delta \bar{\delta} A_{\mu}^{a}, A_{v}^{b}(y)\right\rangle .
$$

In what follows we shall study the structure of the Fourier transforms of these Green's functions.

Let $F_{\mu}$ and $G_{\nu}$ be vector fields and introduce

$$
\left\langle F_{\mu}(x), G_{v}(y)\right\rangle=\frac{-i}{(2 \pi)^{4}} \int d^{4} k e^{i k \cdot(x-y)} T_{\mu v}(k),
$$

and the Fourier transform of $\left\langle F_{\mu}, G_{v}\right\rangle$ is denoted by

$$
T_{\mu v}(k)=\mathrm{FT}\left\langle F_{\mu}, G_{v}\right\rangle .
$$


Then $T_{\mu \nu}$ can be expressed as a linear combination of two covariants:

$$
T_{\mu v}(k)=-\frac{k_{\mu} k_{v}}{k^{2}+i \varepsilon} T_{0}\left(k^{2}\right)-\left(g_{\mu v}-\frac{k_{\mu} k_{v}}{k^{2}+i \varepsilon}\right) T_{1}\left(k^{2}\right) .
$$

Next we introduce two conditions:

Condition 1) Assume $\partial_{\mu} F_{\mu}=0$ and/or $\partial_{\mu} G_{\mu}=0$, then we get

$$
T_{0}\left(k^{2}\right)=T_{0}, \text { constant } .
$$

Condition 2) Assume in addition to the condition 1) that $Q|0\rangle=\langle 0| Q=0$, where

$$
Q=\int d^{3} x F_{0}(x)
$$

then we have

$$
T_{0}=0
$$

Indeed, when this condition is not met we have a broken symmetry and the Nambu-Goldstone boson shows up in the form $T_{0} \neq 0$.

All three terms in Eq. (3.10) satisfy the condition 1) and their Lehmann representations are given as follows. First, by taking account of the antisymmetry between subscripts $\lambda$ and $\mu$, we find

$$
\mathrm{FT}\left\langle F_{\lambda \mu}, A_{v}\right\rangle=-i\left(k_{\lambda} g_{\mu v}-k_{\mu} g_{\lambda v}\right)\left[\frac{R}{k^{2}+i \varepsilon}+\int d m^{2} \frac{\sigma_{1}\left(m^{2}\right)}{k^{2}-m^{2}+i \varepsilon}\right],
$$

so that we obtain

$$
\mathrm{FT}\left\langle\partial^{\lambda} F_{\lambda \mu}, A_{v}\right\rangle=-R \frac{k_{\mu} k_{v}}{k^{2}-i \varepsilon}+\left(k^{2} g_{\mu v}-k_{\mu} k_{v}\right) \int d m^{2} \frac{\sigma_{1}\left(m^{2}\right)}{k^{2}-m^{2}+i \varepsilon} .
$$

Then, thanks to the condition 2), we have in the absence of operator mixing

$$
\mathrm{FT}\left\langle g \tilde{J}_{\mu}, A_{v}\right\rangle=-\left(k^{2} g_{\mu v}-k_{\mu} k_{v}\right) \int d m^{2} \frac{\sigma_{2}\left(m^{2}\right)}{k^{2}-m^{2}+i \varepsilon} .
$$

Finally we have

$$
\mathrm{FT}\left\langle i \delta \bar{\delta} A_{\mu}, A_{v}\right\rangle=-C \frac{k_{\mu} k_{v}}{k^{2}+i \varepsilon}-\left(k^{2} g_{\mu v}-k_{\mu} k_{v}\right) \int d m^{2} \frac{\sigma_{3}\left(m^{2}\right)}{k^{2}-m^{2}+i \varepsilon} .
$$

In what follows we shall study the properties of these integral representations in more detail in QED and QCD.

In QED we have $R=C=1$, so that

$$
\mathrm{FT}\left\langle\partial^{\lambda} F_{\lambda \mu}, A_{v}\right\rangle=-\frac{k_{\mu} k_{v}}{k^{2}+i \varepsilon}+\left(k^{2} g_{\mu v}-k_{\mu} k_{v}\right) \int d m^{2} \frac{\sigma\left(m^{2}\right)}{k^{2}-m^{2}+i \varepsilon},
$$

and

$$
\mathrm{FT}\left\langle-\partial_{\mu} B, A_{v}\right\rangle=-\frac{k_{\mu} k_{v}}{k^{2}+i \varepsilon}
$$


so that we have

$$
\mathrm{FT}\left\langle e \tilde{J}_{\mu}, A_{v}\right\rangle=\left(k^{2} g_{\mu v}-k_{\mu} k_{v}\right) \int d m^{2} \frac{\sigma\left(m^{2}\right)}{k^{2}-m^{2}+i \varepsilon} .
$$

The absence of the massless pole term in (3.21) reflects the fact that the conservation of charge is not broken.

In QCD let us assume that the condition for colour confinement (2.18) is satisfied, then we have

$$
\mathrm{FT}\left\langle\delta \bar{\delta} A_{\mu}, A_{v}\right\rangle=-\left(k^{2} g_{\mu v}-k_{\mu} k_{v}\right) \int d m^{2} \frac{\sigma_{3}\left(m^{2}\right)}{k^{2}-m^{2}+i \varepsilon},
$$

which justifies the absence of the operator mixing in (3.18b). Since $\tilde{J}_{\mu}$ is a linear combination of $J_{\mu}$ and $i \delta \bar{\delta} A_{\mu}$, we can form a linear combination of (3.18b) and (3.22) to find

$$
\mathrm{FT}\left\langle g \tilde{J}_{\mu}, A_{v}\right\rangle=-\left(k^{2} g_{\mu v}-k_{\mu} k_{v}\right) \int d m^{2} \frac{\tilde{\sigma}_{2}\left(m^{2}\right)}{k^{2}-m^{2}+i \varepsilon},
$$

where $\tilde{\sigma}_{2}$ is a linear combination of $\sigma_{2}$ and $\sigma_{3}$. Then substituting (3.18a), (3.22) and (3.23) for Green's functions in Eq. (3.10) we find

$$
R=0,
$$

or

$$
\mathrm{FT}\left\langle\partial^{\lambda} F_{\lambda \mu}, A_{v}\right\rangle=\left(k^{2} g_{\mu v}-k_{\mu} k_{v}\right) \int d m^{2} \frac{\sigma_{1}\left(m^{2}\right)}{k^{2}-m^{2}+i \varepsilon},
$$

Thus we find that there are no contributions to this two-point function from massless gluon states indicating the absence of the massless gluon.

Then what would be the mechanism for generating the finite gluon mass? A possible candidate is the gluon condensate defined by

$$
\left\langle 0\left|A_{\mu}^{a}(x) A_{\mu}^{b}(x)\right| 0\right\rangle=\delta_{a b} K \neq 0
$$

A BRST invariant modification of (3.26) has been considered by Kondo et al to introduce an effective gluon mass [17]. For phenomenological implications of such a gluon condensate as in (3.26), see e.g. [18]. In the Lagrangian density (2.2a) we find a term quartic in the gauge field and replacement of a pair of gauge fields by their vacuum expectation values (3.26) yields a mass term. By keeping only bilinear terms relevant to the description of the free massive gluon field, we find

$$
\mathscr{L}_{g}=\frac{1}{4} A_{a}^{\mu v} A_{\mu \nu}^{a}-\frac{1}{2} m_{0}^{2} A_{a}^{\mu} A_{\mu}^{a},
$$

where

$$
\begin{aligned}
A_{\mu \nu}^{a} & =\partial_{\mu} A_{v}^{a}-\partial_{\nu} A_{\mu}^{a}, \\
m_{0}^{2} & =g_{0}^{2} c_{2}(G) K,
\end{aligned}
$$

where $c_{2}(S U(N))=N$. This gluon condensate does not violate the colour $\mathrm{SU}(3)$ symmetry nor the BRST invariance provided that the mass term in (3.27) is dynamically generated. 


\section{Equivalence Class of Gauges}

In Sec. 2 we have introduced the QCD Lagrangian density that depends on the gauge parameter $\alpha$. We shall study here how theoretical predictions depend on this parameter.

In perturbation theory all the observable quantities are independent of the choice of the gauge parameter, but this is not the case in the non-perturbative approach as we shall see later in this section. Let us consider a class of Lagrangian densities $\left\{\mathscr{L}_{\alpha}\right\}$ representing a gauge theory such as QCD. Assume that all the members of this set are BRST invariant,

$$
\delta \mathscr{L}_{\alpha}=0
$$

and further that the difference between any two elements of this set is exact so that it can be expressed as the BRST transform of a certain operator $\mathscr{M}$,

$$
\Delta \mathscr{L}=\mathscr{L}_{I I}-\mathscr{L}_{I}=\delta \mathscr{M},
$$

then this set $\left\{\mathscr{L}_{\alpha}\right\}$ is called an equivalence class of gauges (ECG) in a loose sense. We shall show latter, however, that we have to introduce an additional condition for identifying the element of an ECG in the non-perturbative approach.

Lagrangian densities corresponding to different choices of $\alpha$ in (2.2) belong to the same class in perturbation theory since we have

$$
\Delta \mathscr{L}=\frac{1}{2}(\Delta \alpha) B \cdot B=-\frac{i}{2}(\Delta \alpha) \delta(\bar{c} \cdot B),
$$

or

$$
\mathscr{M}=-\frac{i}{2}(\Delta \alpha)(\bar{c} \cdot B)
$$

Now we introduce Green's functions in two gauges of the same ECG; then they are related to one another through the Gell-Mann-Low relation [19]:

$$
\left\langle A\left(x_{1}\right) B\left(x_{2}\right) \cdots\right\rangle_{I I}=\left\langle A\left(x_{1}\right) B\left(x_{2}\right) \cdots \exp (i \Delta S)\right\rangle_{I},
$$

where $A, B, \cdots$ are local operators, and

$$
\Delta S=\int d^{4} x \Delta \mathscr{L}=\delta \int d^{4} x \mathscr{M} .
$$

In particular, when all the local operators are BRST invariant, namely

$$
\delta A=\delta B=\cdots=0,
$$

we obtain

$$
\left\langle A\left(x_{1}\right) B\left(x_{2}\right) \cdots\right\rangle_{I I}=\left\langle A\left(x_{1}\right) B\left(x_{2}\right) \cdots\right\rangle_{I},
$$

by expanding the r.h.s of Eq. (4.5) in powers of $\Delta S$. Since $A, B, \cdots$ are closed and $\Delta S$ is exact, we have

$$
\left\langle A, B, \cdots,(\Delta S)^{n}\right\rangle=0 .
$$

Since we are exploiting a series expansion in powers of $\Delta S$, the proof of (4.8) is based on an assumed convergence of this series. 
In what follows we shall restrict ourselves to the Lagrangian density (2.2) and assume that all the quark masses have certain fixed values. Then a theory is characterized by $\alpha$ and $g^{2}$. We shall plot $\left(\alpha, g^{2}\right)$ on a two-dimensional half-plane

$$
-\infty<\alpha<\infty, \quad g^{2}>0,
$$

then each point on this half-plane corresponds to one theory. Now we introduce renormalization group (RG) with the generator of this RG given by [14]

$$
\mathscr{D}=\mu \frac{\partial}{\partial \mu}+\beta(g) \frac{\partial}{\partial g}-2 \alpha \gamma_{V}(g, \alpha) \frac{\partial}{\partial \alpha}
$$

where $\mu$ denotes the renormalization point with dimension of mass and $\gamma_{V}$ is the anomalous dimension of the colour gauge field. Since we have discussed applications of RG to Green's functions elsewhere $[3,14]$, we shall confine ourselves to the study of running parameters defined by

$$
\begin{aligned}
& \bar{g}(\rho)=\exp (\rho \mathscr{D}) \cdot g, \\
& \bar{\alpha}(\rho)=\exp (\rho \mathscr{D}) \cdot \alpha, \\
& \bar{\mu}(\rho)=\exp (\rho \mathscr{D}) \cdot \mu=e^{\rho} \mu,
\end{aligned}
$$

where $\rho$ denotes the parameter of RG. In what follows we shall concentrate our attention on $\bar{g}(\rho)$ and $\bar{\alpha}(\rho)$.

When we increase $\rho$ from 0 to $\infty$, the point $(\bar{\alpha}(\rho), \bar{g}(\rho))$ moves along a line called the RG flow line (RGFL). Two theories corresponding to two points on the same RGFL are completely equivalent and physically identical as has been clarified in the applications of RG to Green's functions $[3,14]$. The asymptotic limits of these running parameters are denoted by

$$
\bar{g}(\infty)=g_{\infty}, \quad \bar{\alpha}(\infty)=\alpha_{\infty},
$$

and such a point represents a sink of the RGFL. Asymptotic freedom that has been assumed throughout this article is characterized by

$$
g_{\infty}=0
$$

It should be emphasized that $\alpha_{\infty}$ can assume only three alternative values depending on the choice of the starting point of the RGFL, namely

$$
\alpha_{\infty}=-\infty, 0, \alpha_{0}
$$

where $\alpha_{0}$ depends only on the number of quark flavors $N_{f}$ in QCD

$$
\alpha_{0}=-\frac{1}{3}\left(13-\frac{4}{3} N_{f}\right)
$$

Without loss of generality we shall restrict ourselves to the following case:

$$
\alpha_{0}>0, \text { or } N_{f}<10 \text {. }
$$


The value of $\alpha_{\infty}$ depends only on the initial value of $\alpha$, namely:

$$
\alpha_{\infty}=\left\{\begin{array}{c}
\alpha_{0}, \text { for } \alpha>0 \\
0, \text { for } \alpha=0 \\
-\infty, \text { for } \alpha<0
\end{array}\right.
$$

We recall the arguments on the ECG. Equivalence of two gauges characterized by $\left(\alpha_{1}, g_{1}^{2}\right)$ and $\left(\alpha_{2}, g_{2}^{2}\right)$ was based on series expansion in powers of $\Delta \alpha$, and when they belong to the same ECG so do also $\left(\bar{\alpha}_{1}, \bar{g}_{1}^{2}\right)$ and $\left(\bar{\alpha}_{2}, \bar{g}_{2}^{2}\right)$. The equivalence of the latter is based on the series expansion in powers of $\Delta \bar{\alpha}(\rho)$. Now assume that $\alpha_{1}<0$ and $\alpha_{2}>0$; however small the difference $\Delta \alpha=\alpha_{2}-\alpha_{1}$ might be, $\alpha_{1}$ tends to $\infty$ and $\alpha_{2}$ to $\alpha_{0}$ so that $\Delta \bar{\alpha}(\rho)$ tends to $\infty$. This fact casts a doubt on the convergence of the power series and we may conclude that these two points do not belong to the same ECG. Thus it is very likely that we have two sets of ECG defined on the two-dimensional parameter half-plane

$$
\begin{gathered}
\mathscr{D}(-\infty)=\left\{\left(\alpha, g^{2}\right): \alpha<0, g^{2}>0\right\} \\
\mathscr{D}\left(\alpha_{0}\right)=\left\{\left(\alpha, g^{2}\right): \alpha>0, g^{2}>0\right\} .
\end{gathered}
$$

Both of them are two-dimensional domains, but in addition we have a one-dimensional line which forms the border between them, namely,

$$
L(0)=\left\{\left(\alpha, g^{2}\right): \alpha=0, g^{2}>0\right\} .
$$

Indeed, $\bar{\alpha}(\rho)$ shows a discontinuity at $\alpha=0$ when $\rho$ is chosen sufficiently large as it is the case for $\alpha_{\infty}$. Then with the help of Eq. (4.9) of [4], i.e.

$$
G\left(p_{i} ; g, \alpha, \mu\right)=\exp \left[\int_{0}^{\rho} d \rho^{\prime} \bar{\gamma}\left(\rho^{\prime}\right)\right] \cdot G\left(p_{i} ; \bar{g}(\rho), \bar{\alpha}(\rho), \bar{\mu}(\rho)\right),
$$

where $\gamma$ denotes the anomalous dimension of the Green's function in question, we may conclude that Green's functions also develop a discontinuity at $\alpha=0$. This discontinuity is certainly reflected in the Green's functions, for instance, in the form of the residue of the massless pole of the gluon propagator studied in the preceding section.

In this connection it is also important to recognize that particle masses defined as the pole positions of propagators are independent of $\rho$ and thus are RG invariant as is clear from the above relationship.

Thus it seems likely that we have three ECG specified by the value of $\alpha_{\infty}$.

It has been shown already that the renormalization constant $Z_{3}$ of the colour gauge field is given by $[3,14]$

$$
Z_{3}^{-1}=\frac{\alpha}{\alpha_{\infty}}
$$

This constant is related to $C$ in (2.19) through the formula [1 - 8]

$$
\bar{C}(\rho)=\bar{a}(\rho)-2 \int_{\rho}^{\infty} d \rho^{\prime}\left(\bar{\gamma}_{V}\left(\rho^{\prime}\right)+\bar{\gamma}_{F P}\left(\rho^{\prime}\right)\right) \bar{a}\left(\rho^{\prime}\right) \exp \left[-2 \int_{\rho}^{\rho^{\prime}} d \rho^{\prime \prime} \bar{\gamma}_{F P}\left(\rho^{\prime \prime}\right)\right],
$$


where $\gamma_{F D}$ is the anomalous dimension of the Faddeev Popov ghost fields and $\bar{a}(\rho)$ is given by

$$
\bar{a}(\rho)=\frac{\bar{\alpha}(\rho)}{\alpha_{\infty}} .
$$

The validity of Eq. (4.23) has been tested in QED with the result that it reproduces $C=1$ correctly and the lack of asymptotic freedom in QED does not allow $C$ to vanish. Hence charge confinement does not take place in QED. Therefore we have

$$
Z_{3}^{-1}= \begin{cases}0, & \text { in } \mathscr{D}(-\infty) \text { and } L(0) \\ \frac{\alpha}{\alpha_{0}}, & \text { in } \mathscr{D}\left(\alpha_{0}\right)\end{cases}
$$

Combining (4.23) and (4.25) we find

$$
\begin{aligned}
& C=0, \text { in } \mathscr{D}(-\infty) \text { and } L(0), \\
& C \neq 0, \text { in } \mathscr{D}\left(\alpha_{0}\right) .
\end{aligned}
$$

This amounts to the conclusion that gluons are massive in the confinement phase, $\mathscr{D}(-\infty)$ and $L(0)$, whereas they remain massless in the deconfinement phase $\mathscr{D}\left(\alpha_{0}\right)$.

The case of $\alpha_{0}<0$ is slightly more complicated, but essential features such as the existence of two phases are the same.

\section{Conclusions}

Theories described by the Lagrangian density (2.1) corresponding to different choices of the gauge parameter $\alpha$ belong to the same ECG in perturbation theory. This class is split into three, however, in non-perturbative approach depending on their convergence properties. Green's functions in QCD are expanded in double power series in $g^{2}$ and $\alpha g^{2}$, and the critical parameters governing the convergence of the expansion are the asymptotic values of $g^{2}, \alpha$, and $\alpha g^{2}$. The last one cannot be evaluated exactly, but is is still possible to judge whether it is equal to zero or non-zero.

In order to clarify the differences among the three classes in the convergence properties we shall tabulate them in what follows:

Case 1) $\alpha_{0}>0$ or $N_{f}<10$.

\begin{tabular}{||l|l|l|l|l||}
\hline \hline region & $\left(\alpha g^{2}\right)_{\infty}$ & $Z_{3}^{-1}$ & confinement & gluon mass \\
\hline$D(-\infty):$ & $\neq 0$ & 0 & yes & $\neq 0$ \\
\hline$D(0)$ or $L(0):$ & 0 & 0 & yes & $\neq 0$ \\
\hline$D\left(\alpha_{0}\right)$ & 0 & $\neq 0, \infty$ & no & 0 \\
\hline \hline
\end{tabular}

In this case $D(0)$ or $L(0)$ is a straight line $\alpha=0$ and forms the border between $D(-\infty)$ and $D\left(\alpha_{0}\right)$. 
Case 2) $\alpha_{0}<0$ or $10 \leq N_{f} \leq 16$.

\begin{tabular}{||l|l|l|l|l||}
\hline \hline region & $\left(\alpha g^{2}\right)_{\infty}$ & $Z_{3}^{-1}$ & confinement & gluon mass \\
\hline$D(-\infty):$ & $\neq 0$ & 0 & yes & $\neq 0$ \\
\hline$D\left(\alpha_{0}\right)$ or $L\left(\alpha_{0}\right):$ & 0 & $\neq 0, \infty$ & no & 0 \\
\hline$D(0)$ & 0 & $\infty$ & no & 0 \\
\hline \hline
\end{tabular}

In this case $D\left(\alpha_{0}\right)$ or $L\left(\alpha_{0}\right)$ is a RGFL and forms the border between $D(-\infty)$ and $D(0)$.

Our naive belief in the independence of physics on the gauge parameter is no longer justified, but instead three phases show up. There are confinement and deconfinement phases, and in the former gluons turn out to be massive and in the latter they remain massless.

Colour confinement is one of the central issues in QCD so that there are various interpretations of this feature. In this paper we have adopted the interpretation that coloured particles are not subject to observation just because coloured states are unphysical in the sense of Eq. (2.16). Then the emergence of massive gluons is an inevitable consequence of this interpretation.

\section{References}

[1] K. Nishijima, Confinement of quarks and gluons, Int. J. Mod. Phys. A 9, 3799 (1994).

[2] K. Nishijima, Confinement of quarks and gluons II, Int. J. Mod. Phys. A 10, 3155 (1995).

[3] K. Nishijima, BRS invariance, asymptotic freedom and color confinement (a review), Czech. J. Phys. 46, 1 (1996).

[4] M. Chaichian and K. Nishijima, Renormalization constant of the color gauge field as a probe of confinement, Eur. Phys. J. C 22, 463 (2001) [hep-th/ 0010079 ].

[5] M. Chaichian and K. Nishijima, Does color confinement imply massive gluons?, Eur. Phys. J. C 47, 737 (2006).

[6] M. Chaichian and K. Nishijima, An Essay on color confinement, in Quantum Field Theory: A Twentieth Century Profile, with a foreword by Freeman J. Dyson, ed. A. N. Mitra, Hindustan Book Agency (India) and Indian National Science Academy, 2000 [hep-th/9909158].

[7] M. Chaichian and K. Nishijima, Significance of the renormalization constant of the colour gauge field, in proceedings of the 2002 International Workshop on Strong Coupling Gauge Theories and Effective Field Theories, Nagoya, Japan, 10-13 December 2002 [hep-th/030220 8].

[8] M. Chaichian and K. Nishijima, The Goto-Imamura-Schwinger term and renormalization group, Sci. Rev. J. 31, 57 (1999) [hep-th/9909159].

[9] D. J. Gross and F. Wilczek, Ultraviolet behavior of non-Abelian gauge theories, Phys. Rev. Lett. 30, 1343 (1973).

[10] H. D. Politzer, Reliable perturbative results for strong interactions?, Phys. Rev. Lett. 30, 1346 (1973).

[11] C. Becchi, A. Rouet and R. Stora, Renormalization of gauge theories, Ann. Phys. 98, 287 (1976); I. V. Tyutin, Gauge invariance in field theory and statistical mechanics, Lebedev preprint FIAN, $\mathrm{n}^{\circ} 39$ (1975). 
[12] K. Nishijima, The Casimir operator in the representations of BRS algebra, Prog. Theor. Phys. 80, 897 (1988).

[13] K. Nishijima, Observable states in the representations of BRS algebra, Prog. Theor. Phys. 80, 905 (1988).

[14] K. Nishijima and N. Takase, Spectral function sum rule for gauge fields, Int. J. Mod. Phys. A 11, 2281 (1996).

[15] R. Oehme and W. Zimmermann, Quark and gluon propagators in quantum chromodynamics, Phys. Rev. D 21, 471, 1661 (1980).

[16] K. Nishijima, Asymptotic conditions and perturbation theory, Phys. Rev. 119, 485 (1960).

[17] K.-I. Kondo, Weak gauge-invariance of dimension two condensate in Yang-Mills theory, Phys. Lett. B 619, 377 (2005) [hep-th/ 0504088$]$;

K.-I. Kondo, A physical meaning of mixed gluon-ghost condensate of mass dimension two, Phys. Lett. B 572, 210 (2003) [hep-th/0306195] and references therein.

[18] F. V. Gubarev, L. Stodolsky and V. I. Zakharov, On the significance of the vector potential squared, Phys. Rev. Lett. 86, 2220 (2001) [hep-ph/ 0010057 ];

F. V. Gubarev and V. I. Zakharov, Emerging phenomenology of $\left\langle A_{\min }^{2}\right\rangle$, Phys. Lett. B 501, 28 (2001) [hep-ph/0010096].

[19] M. Gell-Mann and F. Low, Bound states in quantum field theory, Phys. Rev. 84, 350 (1951). 